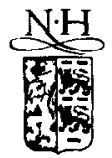

\title{
Cycle-preserving extension of demand functions to new commodities
}

\author{
Hans Peters ${ }^{\mathrm{a}, *}$, Peter Wakker ${ }^{\mathrm{b}}$ \\ ${ }^{a}$ Department of Economics, University of Limburg, P.O. Box 616, 6200 MD Maastricht, Netherlands \\ ${ }^{6}$ Medical Decision Making Unit, University of Leiden, P.O. Box 9600, 2300 RC Leiden, Netherlands
}

Submitted November 1994; accepted July 1995

\begin{abstract}
A method is given to extend demand functions to new commodities under preservation of the cycle number, i.e. the minimal length of a preference cycle revealed by the demand function. Thus, Gale's (Economica, N.S., 1960, 27, 348-354) demand function that shows that the weak axiom of revealed preference does not imply the strong axiom of revealed preference for three commodities can be extended to more than three commodities. Also Shafer's (Journal of Economic Theory, 1977, 16, 293-309) result, that arbitrarily high cycle numbers exist for three commodities, can now be extended to any number of commodities larger than three. This completely settles a question raised by Samuelson (Economica, N.S., 1953, 20, 1-9).
\end{abstract}

JEL classification: C60; D11

Keywords: Revealed preference; WARP; SARP; Preference cycles

\section{Introduction}

Samuelson (1938) showed that the weak axiom of revealed preference (WARP) is necessary for the rationalizability of a demand function by a preference relation. The WARP excludes cycles of length two in the revealed preferences. It has been characterized by Clark (1959, Section 3), Kim and Richter (1986, Section 7), and

\footnotetext{
${ }^{*}$ Corresponding author.
} 
Kehoe (1992, Section 2). An open question remained as to whether or not the WARP was also sufficient for rationalizability. Ville (1946) and Houthakker (1950) showed that the strong axiom of revealed preference (SARP), excluding cycles of any length, was necessary and sufficient. Rose (1958) proved for two commodities that the SARP is implied by the WARP. Gale (1960) demonstrated that this does not hold for three commodities. Peters and Wakker (1994) extended Gale's counterexample to any higher number of commodities, showing that the WARP does not imply the SARP for any number of commodities larger than two.

An open question, raised by Samuelson (1953), was whether exclusion of cycles up to a certain length $k$ would suffice to imply the SARP. A positive answer to this question would give an upper bound to the minimal number of observations needed to refute the SARP. This upper bound $k$ might depend on the number of commodities. For instance, the mentioned result of Rose (1958) shows that for two commodities, $k$ can be taken as equal to 2 . For three commodities, however, Shafer (1977) provided a negative answer to Samuelson's question. That is, he showed that, in that case, such an upper bound $k$ does not exist.

The present paper provides a general method to extend a demand function to more commodities while preserving cycles. Thus, it also preserves the 'cycle number', i.e. the minimal length of a preference cycle revealed by the demand function. As an intermediate tool, this method employs demand functions on nonlinear budget sets, studied in Peters and Wakker (1991).

The method of this paper can be used to extend the counterexamples of Gale (1960) and Shafer (1977) to more than three commodities. It follows that, for any number of commodities higher than two, arbitrarily high cycle numbers can be found, so that the answer to Samuelson's question is negative.

\section{Preparations}

Let $\mathbb{R}_{+}^{n}$ be the set of commodity bundles, $\Sigma^{n}$ the collection of budget sets $B^{n}$ of the form:

$$
B^{n}=\left\{x \in \mathbb{R}_{+}^{n}: p \cdot x \leqslant \alpha\right\},
$$

for some price vector $p \in \mathbb{R}_{++}^{n}$ and income $\alpha \geqslant 0$, and $D^{n}$ a demand function that assigns to each budget set the commodity bundle chosen from the budget set. Choosing the budget set as the argument of the demand function, rather than the vector of prices and income, is more convenient for the purpose of this paper. Throughout, we assume that, for each commodity, there exists a budget set in which a positive amount of that commodity is demanded. Otherwise, the commodity would never be bought and could be suppressed, and the demand function would essentially apply to fewer commodities.

$R$ is the (directly) revealed preference relation, i.e. $x R y$ if there exists a budget set from which $x$ is chosen, whereas $y$ also is contained in the budget set. We 
write $x P y$ if $x R y$ and $x \neq y . P$ is called the directly revealed strict preference relation.

For a subset $T$ of $\mathbb{R}^{n}$ the set:

$$
\operatorname{SE}(T):=\left\{x \in T: \text { there is no } y \in T \text { with } y_{j} \geqslant x_{j} \text { for all } j, y \neq x\right\},
$$

is the (strongly) efficient subset of $T$. Throughout, $D^{n}$ is assumed to satisfy efficiency, i.e. $D^{n}\left(B^{n}\right) \in \operatorname{SE}\left(B^{n}\right)$ for every budget set $B^{n}$.

$D^{n}$ satisfies the SARP if there does not exist a cycle $x^{0} P x^{1} P, \ldots, P x^{k}=x^{0}$, where $k>0$ is the length of the cycle. $D^{n}$ satisfies the WARP if there do not exist cycles of length two.

For a demand function $D^{n}$ that reveals cycles, the 'cycle number' is the minimal length of those cycles. That is, if the cycle number is $k$, then $D^{n}$ reveals a cycle of length $k$, but does not reveal cycles of length smaller than $k$. Thus, at least $k$ choices must be observed to refute the SARP for $D^{n}$. The WARP means that the cycle number is at least three. If the demand function does not reveal cycles, i.e. if it satisfies the SARP, then we say that the cycle number is infinite.

\section{The main result}

This section describes a general method for extending demand functions that satisfy the WARP to more commodities. That is, a continuous efficient demand function $D^{n}$ on $\Sigma^{n}$ is extended to a continuous efficient demand function $D^{m}$ on $\Sigma^{m}(n<m)$ with preservation of the cycle number, which is assumed to be at least three. Subsequently the following theorem can be proved.

Theorem 1. For any number of commodities greater than two:

(a) the WARP does not imply the SARP;

(b) There exist arbitrarily high but finite cycle numbers.

The demand functions $D^{n}$ and $D^{m}$, as well as their cycles, are related in a special way: each cycle of the lower dimensional demand function $D^{n}$ generates a cycle of the same shape for the higher dimensional demand function $D^{m}$ (for details, see Observation 10 in Appendix A). Conversely, every cycle of $D^{m}$ generates a lower dimensional, 'projected' cycle of $D^{n}$.

Next, we describe the construction of $D^{m}$ from $D^{n}$, which involves a number of steps. Proofs are given in Appendix A, as is a small generalization of the construction.

Step 1 . In this step, $m$-dimensional (linear) budget sets are mapped to non-linear $n$-dimensional 'budget sets' by combining commodities $n$ up to $m$ into a single new commodity, as follows. We fix a function $h: \mathbb{R}_{+} \rightarrow \mathbb{R}_{+}$that is surjective, strictly increasing, strictly concave, and continuously differentiable; 
e.g. $h(t)=\ln (t+1)$. We define $f: \mathbb{R}_{+}^{m} \rightarrow \mathbb{R}_{+}^{n}$ by $f:\left(x_{1}, \ldots x_{m}\right) \rightarrow$ $\left(x_{1}, \ldots, x_{n-1}, x_{n}+h\left(x_{n+1}\right)+\ldots+h\left(x_{m}\right)\right)$. The interpretation is that units of the new commodities $n+1, \ldots, m$ are exchanged for units of commodity $n$ at a decreasing exchange rate, described by the function $h$.

Now to each budget set $B^{m} \in \Sigma^{m}$ the lower dimensional set $f\left(B^{m}\right) \subset \mathbb{R}_{+}^{\prime \prime}$ is assigned. The set $f\left(B^{m}\right)$ is convex and compact.

For Steps 2 and 3, we fix a budget set $B^{m} \in \Sigma^{m}$.

Step 2. In the set $f\left(B^{m}\right)$, a unique element, denoted $D^{n}\left(f\left(B^{m}\right)\right)$, can be found, and a linear budget set $B^{n}$, such that:

(i) $B^{n}$ contains the set $f\left(B^{m}\right)$;

(ii) $D^{n}\left(B^{n}\right)=D^{n}\left(f\left(B^{m}\right)\right)$.

Note that $B^{n}$ is tangential to $f\left(B^{m}\right)$ at $D^{n}\left(f\left(B^{m}\right)\right)$. The uniqueness of the element $D^{n}\left(f\left(B^{m}\right)\right)$ follows from the WARP. The proof of this step invokes Brouwer's fixed point theorem, and is given in Appendix $A$.

Step 3. In this step, the demand vector $D^{m}\left(B^{m}\right)$ is constructed. Its first $n-1$ coordinates are taken to be identical to those of $D^{n}\left(f\left(B^{m}\right)\right)$. Then the function $f_{n}$, i.e. $x_{n}+h\left(x_{n+1}\right)+\ldots+h\left(x_{m}\right)$, is maximized over the points in $B^{m}$, with the first $n-1$ coordinates fixed at $D^{n}\left(f\left(B^{m}\right)\right)_{1}, \ldots, D^{n}\left(f\left(B^{m}\right)\right)_{n-1}$. The maximizer is uniquely determined (see Lemma 4 in Appendix $A$ ). $D^{m}\left(B^{m}\right)$ is defined as this maximizer.

The definition of $D^{m}$ has now been completed. Next, we state its relevant properties; these will be derived in Appendix A.

Theorem 2. The demand function $D^{m}$ as constructed above is efficient, continuous, and has the same cycle number as $D^{n}$.

Now we can extend the results of Gale (1960) and Shafer (1977) to more than three commodities, i.e. we can prove Theorem 1.

Proof of Theorem 1. For three commodities. (a) was demonstrated by Gale (1960) and (b) by Shafer (1977). By Theorem 2, these results can be extended to any number of commodities.

\section{Conclusion}

This paper has presented a method to extend demand functions to larger numbers of commodities, while preserving cycles. In this way, a complete answer is obtained to a question raised by Samuelson (1953). This question was whether 
or not exclusion of revealed preference cycles up to a certain length $k$ (possibly depending on the number of commodities) would suffice to imply the SARP. Rose (1958) showed that we can take $k=2$ for the case of two commodities. Shafer (1977) showed that, for three commodities, no such $k$ exists. Using Shafer's examples, we have shown that, for any number of commodities larger than two, no such $k$ exists. This provides a generalization of earlier results by Gale (1960), Shafer (1977), and Peters and Wakker (1994).

\section{Appendix A}

This appendix contains proofs of the results of Section 3. For convenience of presentation, the construction described there will be considered here only for the case $m=n+1$. The general case follows from repeated application of the construction. The argument generalizes that of Peters and Wakker (1994). For the results of the present paper, the number of commodities is assumed to be arbitrary, and the demand function $D^{n}$ need not be surjective. The main additional complication for our generalization lies in the application of Brouwer's theorem: here, a different mapping must be used compared with that of Peters and Wakker (1994).

Convexity and compactness of $f\left(B^{n+1}\right)$ (cf. Step 1) can be proved similarly to Peters and Wakker (1994).

For $x=\left(x_{1}, \ldots, x_{n}\right), y=\left(y_{1}, \ldots, y_{n}\right)$, we write $x \geqslant y$ if $x_{i} \geqslant y_{i}$ for $i=$ $1, \ldots, n ; x \leqslant y$ is similar. We write $x>y$ if $x_{i}>y_{i}$ for $i=1, \ldots, n ; x<y$ is similar. For a subset $T$ of $\mathbb{R}^{n}$ the set:

$$
\operatorname{WE}(T):=\{x \in T: \text { there is no } y \in T \text { with } y>x\},
$$

is the weakly efficient subset of $T$.

Lemma 3. For each budget set $B^{n+1} \in \Sigma^{n+1}, \operatorname{WE}\left(f\left(B^{n+1}\right)\right)=\operatorname{SE}\left(f\left(B^{n+1}\right)\right)$.

Proof. Let $x \in f\left(B^{n+1}\right)$, and $x \leqslant x^{\prime}, x \neq x^{\prime}$ for some $x^{\prime} \in f\left(B^{n+1}\right)$, i.e. $x \notin$ $\operatorname{SE}\left(f\left(B^{n+1}\right)\right)$. Suppose $x_{j}^{\prime}>x_{j}$. We show that $x \notin \mathrm{WE}\left(f\left(B^{n+1}\right)\right)$, by constructing a $\bar{y} \in B^{n+1}$ with $f(\bar{y})>x$. Let $\bar{x}, \bar{x}^{\prime} \in B^{n+1}$ be such that $f(\bar{x})=x, f\left(\bar{x}^{\prime}\right)=x^{\prime}$. We distinguish two cases.

In the first case, $j<n$. Then let $\bar{y}$ be such that $\bar{x}_{j}<\bar{y}_{j}<\bar{x}_{j}^{\prime}$, and, for all $i \neq j$, $\bar{y}_{i}>\bar{x}_{i}^{\prime}$.

In the second case, $j=n$. Then $\bar{x}_{n}^{\prime}>\bar{x}_{n}$ or $\bar{x}_{n+1}^{\prime}>\bar{x}_{n+1}$. Take $\bar{y} \in B^{n+1}$ such that $x_{n}=f_{n}(\bar{x})=\bar{x}_{n}+h\left(\bar{x}_{n+1}\right)<\bar{y}_{n}+h\left(\bar{y}_{n+1}\right)=f_{n}(\bar{y})<f_{n}\left(\bar{x}^{\prime}\right)$ and $\bar{y}_{k} \leqslant \bar{x}_{k}^{\prime}$ for $k=n, n+1$, and $\bar{y}_{k}>\bar{x}_{k}^{\prime} \geqslant \bar{x}_{k}$ for all $k<n$. Then $\bar{y}$ is as required.

The proof of the next lemma is similar to the proof of Lemma 1 in Peters and Wakker (1994), so is omitted. 
Lemma 4. Let $B^{n+1} \in \Sigma^{n+1}, \bar{z}_{1}, \bar{z}_{2}, \ldots, \bar{z}_{n-1} \in \mathbb{R}_{+}$, and suppose that the set $\left\{z \in B^{n+1}: z_{1}=\bar{z}_{1}, z_{2}=\bar{z}_{2}, \ldots, z_{n-1}=\bar{z}_{n-1}\right\}$ is non-empty. Then $f_{n}$ attains its maximum over that set at a unique point.

As in Steps 2 and 3 of Section 3, we now fix a budget set $B^{n+1} \in \Sigma^{n+1}$ determined by prices $\left(p_{1}, \ldots, p_{n+1}\right)$, and income $\alpha$. For every $x \in \operatorname{SE}\left(f\left(B^{n+1}\right)\right)$ let $\left(x_{1}, \ldots, x_{n-1}, \bar{x}_{n}, \bar{x}_{n+1}\right) \in B^{n+1}$ be the unique $f$ original of $x$, cf. Lemma 4. It is also denoted by $f^{-1}(x)$. An explicit expression for the vector $f^{-1}(x)$ can be inferred from the proof of Lemma 6 below.

Lemma $5 . f^{-1}(x)$ is continuous.

Proof. Let $x^{k} \rightarrow x$ in $\operatorname{SE}\left(f\left(B^{n+1}\right)\right)$. By compactness of $B^{n+1}$, we may assume that $f^{-1}\left(x^{k}\right)$ converges to, say, $y$. By continuity of $f, f\left(f^{-1}\left(x^{k}\right)\right) \rightarrow f(y)$, that is, $x^{k}$ converges to $f(y)$. Because $x^{k}$ also converges to $x, f(y)=x$, which, by Lemma 4 , uniquely determines $y$ as $f^{-1}(x)$. Thus, $f^{-1}$ is continuous.

Let the map $N: \operatorname{SE}\left(f\left(B^{n+1}\right)\right) \rightarrow \mathbb{R}^{n}$ be defined by

$$
N(x):=\left(p_{1}, \ldots, p_{n-1}, \min \left\{p_{n}, \frac{p_{n+1}}{h^{\prime}\left(\bar{x}_{n+1}\right)}\right\}\right) .
$$

Lemma 6. For every $x \in \operatorname{SE}\left(f\left(B^{n+1}\right)\right), N(x)$ is a normal vector of a hyperplane supporting $f\left(B^{n+1}\right)$ at $x$.

Proof. For every $x \in \operatorname{SE}\left(f\left(B^{n+1}\right)\right)$, let $c(x):=\alpha-\sum_{i=1}^{n-1} p_{i} x_{i}$.

First, suppose $h^{\prime}(0) \leqslant p_{n+1} / p_{n}$. In this casc, for cvery $x \in \operatorname{SE}\left(f\left(B^{n+1}\right)\right), \bar{x}_{n}=$ $c(x) / p_{n}$ and $\bar{x}_{n+1}=0$, because these values maximize $f_{n}$ given $\left(x_{1}, \ldots, x_{n-1}\right)$. In words, the marginal contribution to $f_{n}$ of the $n$th commodity is larger than that of the $(n+1)$ th commodity. Therefore, we have

$$
f^{-1}(x)=\left(x_{1}, \ldots, x_{n-1}, c(x) / p_{n}, 0\right),
$$

and

$$
f\left(B^{n+1}\right)=\left\{x \in \mathbb{R}_{+}^{n}:\left(x_{1}, \ldots, x_{n}\right) \cdot\left(p_{1}, \ldots, p_{n}\right) \leqslant \alpha\right\} .
$$

The desired result now follows, because $p_{n+1} / h^{\prime}\left(\bar{x}_{n+1}\right) \geqslant p_{n+1} / h^{\prime}(0) \geqslant p_{n}$ for every $x \in \operatorname{SE}\left(f\left(B^{n+1}\right)\right)$.

Next, suppose $h^{\prime}(0)>p_{n+1} / p_{n}$. We distinguish two cases.

In the first case, consider $x \in \operatorname{SE}\left(f\left(B^{n+1}\right)\right)$ with $h^{\prime}\left(c(x) / p_{n+1}\right) \geqslant p_{n+1} / p_{n}$. Then, given $\left(x_{1}, \ldots, x_{n-1}\right), f_{n}$ is maximized by $\bar{x}_{n}=0$ and $\bar{x}_{n+1}=c(x) / p_{n+1}$. In words, the marginal contribution to $f_{n}$ of the $(n+1)$ th commodity is larger 
than that of the $n$th commodity, even if the remaining 'income' $c(x)$ is completely spent on the $(n+1)$ th commodity. Therefore, in this case, we have

$$
f^{\prime}(x)=\left(x_{1}, \ldots, x_{n-1}, 0, c(x) / p_{n+1}\right) .
$$

Such points $x$ satisfy the equation $G(x)=0$ with $G(x)=p_{n+1} h^{-1}\left(x_{n}\right)-c(x)$. The gradient of $G$ at $x$ is the vector $\left(p_{1}, \ldots, p_{n-1}, p_{n+1} / h^{\prime}\left(h^{-1}\left(x_{n}\right)\right)\right)$, which is equal to $N(x)$, since $p_{n+1} / h^{\prime}\left(h^{-1}\left(x_{n}\right)\right)=p_{n+1} / h^{\prime}\left(\bar{x}_{n+1}\right) \leqslant p_{n}$. Since the equation $G(x)=0$ locally describes $\operatorname{SE}\left(f\left(B^{n+1}\right)\right)$, the convexity of $f\left(B^{n+1}\right)$ implies that $N(x)$ is normal to a supporting hyperplane of $f\left(B^{n+1}\right)$ at $x$.

In the second case, consider $x \in \operatorname{SE}\left(f\left(B^{n+1}\right)\right)$ with $h^{\prime}\left(c(x) / p_{n+1}\right)<p_{n+1} / p_{n}$. Then $\bar{x}_{n+1}$ solves $h^{\prime}\left(\bar{x}_{n+1}\right)=p_{n+1} / p_{n}$ and $\bar{x}_{n}=\left(c(x)-p_{n+1} \bar{x}_{n+1}\right) / p_{n}$. Such points $x$ satisfy $G(x)=0$ where now $G(x)=-c(x)+p_{n} x_{n}+p_{n+1} \bar{x}_{n+1}-$ $p_{n} h\left(\bar{x}_{n+1}\right)$. The gradient of this function $G$ is $\left(p_{1}, \ldots, p_{n}\right)$, which equals $N(x)$, because $p_{n}=p_{n+1} / h^{\prime}\left(\bar{x}_{n+1}\right)$. Therefore, also in this case, $N(x)$ is normal to a supporting hyperplane of $f\left(B^{n+1}\right)$ at $x$.

Next we describe the construction of $D^{n+1}$ from $D^{n}$ in detail. Recall that $D^{n}$ satisfies the WARP. The construction described here is slightly more general than that in Section 3. By this generalization, cycles of $D^{n}$ give rise to cycles of $D^{n+1}$, where positive amounts of each commodity, in particular of the new commodity, are bought. This precludes trivial cases that are essentially lower-dimensional. Further, it enables us to adapt our results to $\mathbb{R}_{++}^{n}$ and $\mathbb{R}_{++}^{m}$ instead of $\mathbb{R}_{+}^{n}$ and $\mathbb{R}_{+}^{m}$; the interest of this has been discussed in Peters and Wakker (1991). For the method described in Section 3, one can take $\mu=0$ below, and make minor adaptations in the subsequent analysis.

To allow restriction to positive coordinates, we assume that there exists $\mu>0$ such that all commodity bundles that occur in cycles of $D^{n}$ have all coordinates greater than or equal to $\mu$. If this assumption is violated, then $D^{n}$ is transformed to $D^{\prime}$ as follows. A value $\mu>0$ is chosen. Then, if a budget set $B^{n}$ has a non-empty intersection $B^{\prime}$ with $[\mu, \infty]^{n}$, then

$$
D^{\prime}\left(B^{n}\right)=D\left(\left(B^{\prime}-(\mu, \ldots, \mu)\right) \cap \mathbb{R}_{+}^{n}\right)+(\mu, \ldots, \mu) ;
$$

otherwise, $D^{\prime}\left(B^{n}\right)$ is the intersection of $B^{n}$ with the line segment that connects the origin and $(\mu, \ldots, \mu)$. Then $D^{\prime}$ contains all cycles of $D^{n}$ shifted by $(\mu, \ldots, \mu)$, and $D^{\prime}$ does not contain other cycles. This is similar to footnote 1 in Peters and Wakker (1994).

Let $x \in \operatorname{SE}\left(f\left(B^{n+1}\right)\right)$, and let $H(x)$ denote the budget set in $\Sigma^{n}$ determined by the hyperplane supporting $f\left(B^{n+1}\right)$ at $x$ with normal $N(x)$. In view of Lemma $6, H(x)$ is well defined; $H$ depends continuously on $x$, since $N$ does, in view of Lemma 5. Let $\psi$ denote projection on $\operatorname{SE}\left(f\left(B^{n+1}\right)\right)$; i.e. $\psi$ assigns to each point the nearest point of $\operatorname{SE}\left(f\left(B^{n+1}\right)\right)$, according to Euclidean distance. Obviously, $\psi$ is continuous, so the map $\psi \circ D^{n} \circ H: \operatorname{SE}\left(f\left(B^{n+1}\right)\right) \rightarrow \operatorname{SE}\left(f\left(B^{n+1}\right)\right)$ is continuous. Since, as a consequence of Lemma 3 , the $\operatorname{set} \operatorname{SE}\left(f\left(B^{n+1}\right)\right)$ is homeomorphic 
to a compact convex set (e.g. the projection on the plane $x_{1}=0$ ), we can invoke Brouwer's theorem and obtain the existence of a point $x^{*} \in \operatorname{SE}\left(f\left(B^{n+1}\right)\right)$ with $x^{*}=\psi \circ D^{n} \circ H\left(x^{*}\right)$. (The budget set $H\left(x^{*}\right)$ is the set $B^{n}$ in Step 2 of Section 3.) We use the fact that, for all $x \in H\left(x^{*}\right) ; \psi(x)=x^{*} \Leftrightarrow x=x^{*}$, applied to $x=D^{n}\left(H\left(x^{*}\right)\right)$, to conclude that $D^{n}\left(H\left(x^{*}\right)\right)=x^{*}$. By the WARP of $D^{n}$, this fixed point $x^{*}$ is unique. Therefore, we can define $D^{n}\left(f\left(B^{n+1}\right)\right)=x^{*}$. Finally, for each $B^{n+1} \in \Sigma^{n+1}$, we let

$$
D^{n+1}\left(B^{n+1}\right):=f^{-1}\left(D^{n}\left(f\left(B^{n+1}\right)\right)\right) \text {. }
$$

This completes the derivation of Steps 2 and 3 in Section 3.

The following three lemmas prove Theorem 2 .

Lemma 7. $D^{n+1}$ is efficient and continuous.

Proof. It is obvious that $D^{n+1}$ is efficient. For continuity, let $B^{n+1}, B_{1}^{n+1}$, $B_{2}^{n+1}, \ldots$, be a sequence in $\Sigma^{n+1}$ with $\lim _{j \rightarrow \infty} B_{j}^{n+1}=B^{n+1}$. Peters and Wakker (1994, Section 4) show that $f$ is continuous with respect to the Hausdorff metric; hence, $f\left(B_{j}^{n+1}\right)$ converges to $f\left(B^{n+1}\right)$. Each set $f\left(B_{j}^{n+1}\right)$ is supported at the point $D^{n}\left(f\left(B_{j}^{n+1}\right)\right)$ by a hyperplane with positive normal, determining a budget set $B_{j}^{n}$ in $\Sigma^{n}$. By compactness, we may assume that the points $D^{n}\left(f\left(B_{j}^{n+1}\right)\right)$ and sets $B_{j}^{n}$ converge to a point $y$ and a budget set $B^{n}$ respectively. It straightforwardly follows that $B^{n}$ supports $f\left(B^{n+1}\right)$ at $y$. By continuity of $D^{n}$ on $\Sigma^{n}, y=D^{n}\left(B^{n}\right)$. By the definition of $D^{n}\left(f\left(B^{n+1}\right)\right), y$ must be this point $D^{n}\left(f\left(B^{n+1}\right)\right)$. Thus, $D^{n}\left(f\left(B_{j}^{n+1}\right)\right)$ converges to $D^{n}\left(f\left(B^{n+1}\right)\right)$.

We must show that $f^{-1}\left(D^{n}\left(f\left(B_{j}^{n+1}\right)\right)\right) \rightarrow f^{-1}\left(D^{n}\left(f\left(B^{n+1}\right)\right)\right)$ as $j \rightarrow \infty$. Note that the function $f^{-1}$, used above, is different for different sets $B_{j}^{n+1}$, or $B^{n+1}$. This dependence is not expressed in the notation. For this reason, we cannot invoke Lemma 5. Therefore, we use the explicit expressions for $f^{-1}$ given in the proof of Lemma 6 . As the sets $B_{j}^{n+1}$ converge to $B^{n+1}$, so do the associated price vectors, and incomes $\alpha$. Therefore, if $h^{\prime}(0) \leqslant p_{n+1} / p_{n}$, then the desired convergence follows. If $h^{\prime}(0)>p_{n+1} / p_{n}$ and $h^{\prime}\left(c(y) / p_{n+1}\right)>p_{n+1} / p_{n}$, then, for $j$ sufficiently large, the same case applies for the sets $B_{j}^{n+1}$ and the desired convergence follows. Similarly, if $h^{\prime}(0)>p_{n+1} / p_{n}$ and $h^{\prime}\left(c(y) / p_{n+1}\right)<p_{n+1} / p_{n}$, then, for $j$ sufficiently large, the same case applies again for the sets $B_{j}^{n+1}$ and the desired convergence again follows. Finally, if $h^{\prime}(0)>p_{n+1} / p_{n}$ and $h^{\prime}\left(c(y) / p_{n+1}\right)$ $=p_{n+1} / p_{n}$, then both the formulas for the first case in the proof of Lemma 6 and for the second case there can be used, leading to the same result. The desired convergence again follows, both for the sets $B_{j}^{n+1}$ for the first case there, and for the second case there. Thus, we conclude $\lim _{j \rightarrow \infty} D^{n+1}\left(B_{j}^{n+1}\right)=D^{n+1}\left(B^{n+1}\right)$ in all cases.

Lemma 8. The cycle number of $D^{n+1}$ is greater than or equal to the cycle number of $D^{n}$. 
Proof. Let $x^{0}, \ldots, x^{k}$ be a cycle revealed by $D^{n+1}$ through the budget sets $B_{1}^{n+1}, \ldots, B_{k}^{n+1}\left(x^{0}=x^{k}\right)$. Then, in view of (1), $f\left(x^{0}\right) P f\left(x^{1}\right) P \ldots P f\left(x^{k}\right)$ is revealed by budget sets supporting $f\left(B_{1}^{n+1}\right), \ldots, f\left(B_{k}^{n+1}\right)$ at $f\left(x^{0}\right), \ldots, f\left(x^{k-1}\right)$. This yields a cycle of length $k$ or smaller for $D^{n}$ (the length may be smaller if points coincide).

The following lemma extends Lemma 3 in Peters and Wakker (1994).

Lemma 9. The cycle number of $D^{n+1}$ is smaller than or equal to the cycle number of $D^{n}$.

Proof. Fix $z_{n+1}>0$ so small that $h\left(z_{n+1}\right)<\mu$, where $\mu>0$ is a lower bound for coordinates of commodity bundles that occur in cycles of $D^{n}$. Let $f$ be the restriction of $f$ to $\mathbb{R}_{+}^{n} \times\left\{z_{n+1}\right\}$; it is an affine bijection from $\mathbb{R}_{+}^{n} \times\left\{z_{n+1}\right\}$ to $\mathbb{R}_{+}^{n}+\left(0, \ldots, 0, h\left(z_{n+1}\right)\right)$. Let the constants $h_{n+1}^{\prime}$ and $h_{n+1}(0)$ be such that $h_{n+1}\left(x_{n+1}\right)=h_{n+1}^{\prime} x_{n+1}+h_{n+1}(0)$ is the affine function tangential to $h$ at $z_{n+1}$. Let $f:\left(x_{1}, \ldots, x_{n+1}\right) \mapsto\left(x_{1}, \ldots, x_{n-1}, x_{n}+h_{n+1}\left(x_{n+1}\right)\right)$; this function is affine. Let $x^{1} P x^{2}$ be revealed by a linear budget set $B^{n} \subset \mathbb{R}_{+}^{n}$, with all coordinates of $x^{1}$ and $x^{2}$ at least $\mu$. Then

Let $\left(p_{1}, \ldots, p_{n}\right)$ and $\alpha$ be the price vector and income that correspond to $B^{n}$.

$$
\begin{aligned}
& (\bar{f})^{-1}\left(B^{n}\right) \\
& =\left\{\left(x_{1}, \ldots, x_{n+1}\right) \in \mathbb{R}_{+}^{n+1}: p_{1} x_{1}+\ldots+p_{n} x_{n}+p_{n} h_{n+1}\left(x_{n+1}\right) \leqslant \alpha\right\} \\
& =\left\{\left(x_{1}, \ldots, x_{n+1}\right) \in \mathbb{R}_{+}^{n+1}: p_{1} x_{1}+\ldots+p_{n} x_{n}+p_{n} h_{n+1}^{\prime} x_{n+1}\right. \\
& \left.\quad \leqslant \alpha-p_{n} h_{n+1}(0)\right\} .
\end{aligned}
$$

On $\mathbb{R}_{+}^{n} \times\left\{z_{n+1}\right\}, \vec{f}=f=f_{z}$, so $f_{z}^{-1}\left(x^{1}\right), f_{z}^{-1}\left(x^{2}\right) \in(\bar{f})^{-1}\left(B^{n}\right)$; note that $f_{z}^{-1}\left(x^{1}\right)$ and $f_{z}^{-1}\left(x^{2}\right)$ exist because $h\left(z_{n+1}\right)$ is smaller than $x_{n}^{1}$ and $x_{n}^{2}$. Elsewhere, $\bar{f} \geqslant f$. Hence, for each $y \in(\bar{f})^{-1}\left(B^{n}\right), f(y) \geqslant f(y)$; since $f(y)$ is an element of $B^{n}$, so is $f(y)$; thus, $f\left((\bar{f})^{-1}\left(B^{n}\right)\right) \subset B^{n}$. Because $f\left((\bar{f})^{-1}\left(B^{n}\right)\right)$ contains $f\left(f^{-1}\left(x^{1}\right)\right)=$ $x^{1}$, it contains $D^{n}\left(B^{n}\right)=x^{1}$. Therefore, by the WARP, $x^{1}=D^{n}\left(f\left((\tilde{f})^{-1}\left(B^{n}\right)\right)\right)$. It follows by (1) that $D^{n+1}\left((\bar{f})^{-1}\left(B^{n}\right)\right)=f_{z}^{-1}\left(x^{1}\right)$. Therefore, the linear $(n+1)$ dimensional budget set $(\bar{f})^{-1}\left(B^{n}\right)$, which also contains $f_{z}^{-1}\left(x^{2}\right)$, has revealed $f_{z}^{-1}\left(x^{1}\right) P f_{z}^{-1}\left(x^{2}\right)$.

It follows that cycles of $D^{n}$ are mapped by $f_{i}^{-1}$ to cycles of the same shape and length for $D^{n+1}$.

The above proof has also demonstrated the following isomorphism between cycles of $D^{n}$ and of $D^{n+1}$. 
Observation 10. Let $x^{0} P x^{1} P \ldots P x^{k}$ be a revealed preference cycle of $D^{n}$. Take any $z_{n+1}$ such that $x_{n}^{j} \geqslant h\left(z_{n+1}\right)$ for all $j$. Then we obtain an isomorphic revealed preference cycle $y^{0} P y^{1} P \ldots P y^{k}$ of $D^{n+1}$, where, for each $j$ :

$$
y^{j}=\left(x_{1}^{j}, \ldots, x_{n-1}^{j}, x_{n}^{j}-h\left(z_{n+1}\right), z_{n+1}\right) .
$$

The proof also shows that the price vectors used in the two revealed preference cycles are closely related. It can be demonstrated that the adopted budget sets are also isomorphic in a special way. Details are omitted.

\section{References}

Clark, S.A., 1959, An Extension Theorem for rational choice functions, Review of Economic Studies $55,485-492$.

Gale, D., 1960, $\wedge$ note on revealed preference, Economica, N.S. 27, $348 \quad 354$.

Houthakker, H.S., 1950, Revealed preference and the utility function, Economica, N.S. 17, 159-174.

Kehoe, T.J., 1992, Gross substitutability and the weak axiom of revealed preference, Journal of Mathematical Economics 21, 37-50.

Kim, T. and M.K. Richter, 1986, Nontransitive-nontotal consumer theory, Joumal of Economic Theory $38,324-363$.

Peters, H.J.M. and P.P. Wakker, 1991, Independence of irrelevant alternatives and revealed group preferences, Econometrica 59, 1787-1801.

Peters, H.J.M. and P.P. Wakker, 1994, WARP does not imply SARP for more than two commodities, Joumal of Economic Theory 62, 152-160.

Rose, H., 1958, Consistency of preference: The two-commodity case, Review of Economic Studies 35, 124-125.

Samuelson, P.A., 1938, A note on the pure theory of consumer's behaviour, Economica, N.S. 5, $61-71,353-354$.

Samuelson, P.A., 1953, Consumption theorems in terms of over-compensation rather than indifference comparisons, Economica, N.S. 20, 1-9.

Shafer, W.J., 1977, Revealed preference cycles and the Slutsky matrix, Joumal of Economic Theory 16, 293-309.

Ville, J., 1946, Sur les conditions d'existence d'une ophélimité totale et d'un indice du niveau des prix, Annales de l'Université de Lyon 9, Sec. A, no. 3, 32-39. Translated into English by P.K. Newman, 1951-1952. The existence-conditions of a total utility function, Review of Economic Studies 19, $123-128$. 